\title{
Abordaje periodístico de la obesidad en la prensa colombiana durante los años $2008-2013$
}

\author{
Journalistic approach of obesity in the Colombian press during the years 2008 - 2013
}

\author{
María Camila Correa-Madrid', Isabel Cristina Carmona-Garcés², Laura Inés González Zapata ${ }^{3}$ \\ 1 Nutricionista Dietista. Escuela de Nutrición y Dietética, Universidad de Antioquia. Medellín, Colombia. e-mail: \\ mcdaliniana@gmail.com \\ 2 Nutricionista Dietista, Magíster en Educación. Profesora Escuela de Nutrición y Dietética, Universidad de Antioquia. Medellín, \\ Colombia. e-mail: isabel.carmona@udea.edu.co orcid.org/0000-0002-9757-7054 \\ 3 Nutricionista Dietista, Magíster en Epidemiología, PhD Salud Pública. Docente Titular. Grupo de Investigación en \\ Determinantes Sociales de la Salud y la Nutrición, Escuela de Nutrición y Dietética, Universidad de Antioquia. Medellín, \\ Colombia. e-mail: laura.gonzalez@udea.edu.co
}

Fecha de recepción: Junio 24 - $2016 \quad$ Fecha de revisión: Noviembre 01 - $2016 \quad$ Fecha de aceptación: Diciembre 12 - 2016

Correa-Madrid MC, Carmona-Garcés IC, González Zapata LI. Abordaje periodístico de la obesidad en la prensa colombiana durante los años 2008 - 2013. Univ. Salud. 2016;18(3):566-575. DOI: http://dx.doi.org/10.22267/rus.161803.62

\section{Resumen}

Introducción: Una de las funciones de los medios de comunicación masiva es movilizar la sociedad y la agenda política en torno a problemáticas públicas como por ejemplo la obesidad. Objetivo: Describir el abordaje dado al tema obesidad en dos periódicos colombianos durante los años 2008-2013. Materiales y metodos: Estudio transversal, se seleccionaron 163 noticias, 48 del periódico El Espectador y 115 de El Colombiano. Se realizó análisis deductivo, posteriormente el análisis descriptivo mediante pruebas $\mathrm{X}^{2}$ para establecer la relación entre las variables. $\alpha=0,05$. SPSS v.18. Resultados: Se evidenció un aumento gradual de las noticias relacionadas con la obesidad en el periodo estudiado. Las fuentes de información más consultadas para construir las noticias fueron científicas-académicas (42,3\%). Se encontró asociación entre los artículos que tratan la magnitud de la obesidad, sus estrategias para afrontarla y cuando se empleó como fuente a los políticos $(\mathrm{p}<0.05)$. En la población infantil, los temas publicidad $(16,4 \%)$ y comidas rápidas $(26,9 \%)$ tuvieron una mayor cobertura $(\mathrm{p}<0.05)$. Conclusión: La cobertura del tema obesidad creció en Colombia, al mismo tiempo que se empezaron a implementar estrategias para su prevención y control. Esta situación puede favorecer la movilización pública y política frente a nuevas estrategias que propendan por la solución de esta problemática.

Palabras clave: Obesidad; prensa; medios de comunicación; comunicación en salud. (Fuente: DeCS, Bireme).

\begin{abstract}
Introduction: One of the functions of the mass media is to mobilize society and the political agenda around public issues such as obesity. Objective: To describe the approach given to obesity in two Colombian newspapers during the years 2008-2013. Materials and methods: A cross-sectional study was conducted were 163 news were selected from two newspapers; 48 from El Espectador newspaper and 115 from El Colombiano. First a deductive analysis of the news was performed and then a descriptive analysis using $\mathrm{X}^{2}$ tests to establish the relationship between the variables. $\alpha<0.05$. SPSS v.18. Results: An increase in obesity-related news was found. The sources of information most frequently
\end{abstract}


consulted to build the news were scientific-academic ones (42.3\%). It was found an association between the articles dealing with the magnitude of the obesity, the strategies to confront it and when it was used a politician source ( $p$ $<0.05)$. In the pediatric population, issues related with advertising $(16.4 \%)$ and fast food $(26.9 \%)$ had greater coverage $(\mathrm{p}<0.05)$. Conclusion: The mass media news related with obesity increased in Colombia, at the same time the implementation of strategies for prevention and control of this disease started. This situation can foster public and political mobilization that promote new strategies that contribute to the solution of the obesity problem.

Keywords: Obesity; press; mass media; health communication. (Source: DeCS, Bireme).

\section{Introducción}

El aumento continuo de peso corporal de la población, es para el mundo una prioridad en salud en todas sus esferas. El NCD Risk Factor Collaboration (NCD -Risk), con base en un estudio poblacional publicado en 2016, concluyó que si la tendencia post-2000 continúa, la probabilidad de cumplir la meta mundial de reducción de la obesidad es prácticamente nula y que por el contrario a 2025, la prevalencia mundial de obesidad alcanzará el $18 \%$ en los hombres y superará el $21 \%$ en mujeres y la obesidad mórbida superará el $6 \%$ en los hombres y el $9 \%$ en las mujeres ${ }^{1}$. Colombia no es ajena a esta problemática, según la Encuesta Nacional de la Situación Nutricional (ENSIN 2010), el 51,2\% de la población adulta (18-64 años) presenta exceso de peso, de este porcentaje $34,6 \%$ en sobrepeso y $16,5 \%$ en obesidad ${ }^{2}$.

Las Guías colombianas para el manejo científico de la obesidad y el sobrepeso retoman la definición de obesidad de la Organización Mundial de la Salud (OMS), como un incremento en el porcentaje de grasa corporal, acompañado del aumento del peso corporal, cuyo monto y distribución alteran la salud del individuo afectado $^{3}$. Esta acumulación de tejido adiposo suele ser el resultado de un desequilibrio entre las calorías ingeridas y las calorías gastadas ${ }^{4,5}$, sin embargo, son muchos los factores determinantes de esta condición entre ellos genéticos, fisiológicos, sicológicos, ambientales, sociales, económicos y políticos 5,6 , razón por la cual se define como una condición multicausal.

Las consecuencias de la obesidad y el sobrepeso para la salud son diversas, entre ellas sobresalen la enfermedad cardiovascular, hipertensión, diabetes, dislipidemia, osteoartritis, apnea del sueño, síndrome metabólico y algunos tipos de cáncer ${ }^{4}$. De igual manera, en el ámbito económico la obesidad y sus comorbilidades son causa de altos costos para los sistemas de salud ${ }^{7}$.

Las estrategias para la prevención y control de este fenómeno son necesarias y se requiere de acciones del individuo, sociales y contextuales ${ }^{4}$. A partir de la Estrategia Mundial sobre Régimen Alimentario, Actividad Física y Salud, aprobada por la Asamblea Mundial de la Salud en el año 2004, en diversos países se viene implementando acciones con el objetivo de promover y proteger la salud, orientando la creación de un entorno favorable tanto a nivel personal como comunitario $^{8}$. Bajo los lineamientos de la OMS, se promulgó en Colombia la Ley 1355 de 2009, la cual estableció la obesidad y las enfermedades crónicas no transmisibles asociadas a esta, como una prioridad de salud pública y se adoptó medidas para su control, atención y prevención 9 . En consecuencia, la obesidad debería ser uno de los temas principales en la agenda de salud pública de país y un tema fundamental de la agenda mediática en salud.

La agenda mediática juega un rol importante en la configuración de la opinión pública, debido a que puede determinar la presencia y el énfasis que se le dé a un tema dentro de los medios de comunicación ${ }^{10}$, esto sumado a la concepción actual de comunicación en salud, como un componente vital para la práctica de la salud pública, que podría facilitar la difusión de conocimientos, reforzar hábitos, conductas, valores, normas sociales y estimular procesos de cambio social en beneficio de la calidad de vida de las poblaciones ${ }^{11}$.

La OMS define la comunicación en salud como "el estudio y el uso de estrategias de comunicación 
para informar e influir decisiones individuales $y$ comunitarias que mejoren la salud ${ }^{12}$. Los medios de comunicación son una gran herramienta de salud pública, pues no sólo cumplen un papel informativo, sino también de difusión de información a través de los espacios publicitarios, programas de información y opinión, que influyen en los patrones de conducta, valores y creencias de las poblaciones ${ }^{13,14}$. Además, los medios pueden dirigir su atención sobre temas específicos, los cuales durante determinado periodo de tiempo hacen presencia y logran movilizar la opinión pública ${ }^{15}$. Por lo tanto, los conocimientos y opiniones que tengan las poblaciones sobre un tema, obedecerán en parte a la cobertura periodística que se le dedique y al tipo información que se difunda ${ }^{16}$.

Al mismo tiempo, los medios de comunicación audiovisuales, como la prensa y la radio, determinan modelos de comportamiento y actitudes, los cuales le sugieren a los diferentes públicos cómo actuar en diferentes ámbitos ${ }^{17}$. No obstante, los medios de comunicación pueden transmitir información poco ajustada a la realidad científica sobre temas como el sobrepeso y la obesidad ${ }^{18}$.

En Colombia, los medios más consumidos son la televisión, la radio y las revistas independientes; la prensa ocupa el cuarto lugar con un $35,0 \%$ en la estructura de medios, esta cifra se ha mantenido estable entre $27,0 \%$ y $35,0 \%$ desde el año 2007 hasta el $2011^{11}$, sin verse afectada por los nuevos medios de comunicación disponibles.

Desde nuestro conocimiento son escasos los trabajos que tratan el análisis de la cobertura periodística en Colombia sobre temas de sobrepeso y obesidad, sin embargo, investigaciones realizadas en España ${ }^{20,21}$, denotan la importancia de la prensa, como parte de la estrategia de salud pública frente a la problemática. Este trabajo pretende describir el abordaje dado al tema de la obesidad en prensa colombiana y su caracterización en aspectos tales como frecuencia de publicación, sección del periódico en la que aparecen los artículos, población de referencia, temática y las fuentes de información empleadas en la construcción de la noticia.

\section{Materiales y métodos}

Se realizó un estudio descriptivo sobre el abordaje de la temática: obesidad en prensa colombiana durante los años 2008-2013. Para la selección de los periódicos a analizar, se usó el Estudio General de Medios ${ }^{22,23}$, que informa sobre los periódicos más leídos en su sitio de internet, en su orden: El Colombiano, El Tiempo y El Espectador, se descartó el periódico El Tiempo debido a que su buscador no permitió filtrar de manera efectiva las noticias a partir de la palabra "obesidad", lo cual impidió delimitar la búsqueda y llegar al tema de interés.

Los artículos fueron localizados a través del buscador disponible en cada una de las Web de los dos periódicos seleccionados, se usó como palabra clave "obesidad" y los años de interés para la investigación 2008-2013. Se excluyeron las noticias en formato multimedia como videos e imágenes y a partir de la lectura estructural de cada uno de los artículos se descartaron aquellos en los cuales el tema principal no era la obesidad.

Las noticias se analizaron usando el método deductivo, las variables de interés fueron: año de publicación, día de publicación (semana o fin de semana), tipo de reportaje (artículo o columna), sección (noticias, opinión, economía, deportes, salud, columnista, otra), población a la cual hace referencia la noticia (niños, adolescentes, adultos, adultos mayores, población general), fuente de información (científica o académica, médico o especialistas médicos, nutricionista dietista, industria alimentaria y farmacéutica, asociaciones profesionales, sector político, jurídico, organizaciones internacionales, periodista, datos epidemiológicos y de salud pública) y tema específico según las siguientes categorías:

1. Magnitud del problema: su tema principal fueron datos epidemiológicos sobre la obesidad en cualquier escenario.

2. Denuncias: individuos, grupos, asociaciones o agremiaciones hicieron denuncias sobre la 
obesidad o sus factores asociados como un problema.

3. Estética: relacionaron la obesidad con tratamientos estéticos o plásticos.

4. Recomendaciones: sugerencias para perder peso, cambiar hábitos, probar productos o medicamentos nuevos.

5. Gasto sanitario: informes relacionados con el gasto económico para el sistema de salud y la obesidad.

6. Resultados de investigación: avances científicos relacionados con la obesidad y sus factores asociados

7. Publicidad: textos que relacionaron la publicidad con la epidemia de la obesidad.

8. Bebidas azucaradas: referencia a estos productos y su contenido nutricional.

9. Globalización y su relación con la obesidad.

10. Comida rápida: referencia a estos productos y su contenido nutricional.

11. Estrategias: acciones generadas para controlar o minimizar el problema de la obesidad.

Para la recolección de datos se diseñó una ficha que incluyó las variables de interés, la cual fue evaluada y ajustada con los resultados obtenidos en una prueba piloto en la que se analizaron veinte noticias. La recolección y codificación de los datos, se llevó a cabo por el primer autor. Las noticias seleccionadas se clasificaron en una o varias poblaciones, lo mismo para las variables fuente de información y temas. El análisis estadístico se realizó en el software SPSS Versión 18.
El análisis descriptivo de las variables, incluyó el cálculo de frecuencias, la prueba de Chi cuadrado o la prueba exacta de Fisher según el caso, para identificar la relación entre las variables de interés. Se asumió un error alpha de 0,05.

\section{Consideraciones éticas}

Esta investigación se clasificó como “investigación sin riesgo", según la Resolución № 008430 artículo 11 de 1993.

\section{Resultados}

Se revisó un total de 495 noticias sobre obesidad publicadas entre el año 2008 y 2013, 158 en El Espectador y 337 en El Colombiano. De estas publicaciones se excluyeron 332 debido a que el tema principal de la noticia no era la obesidad o estaban en formato multimedia, resultando una muestra final de163 noticias 48 del periódico El Espectador y 115 de El Colombiano. La frecuencia de publicación mostró un aumento gradual de las noticias relacionadas con la obesidad durante el periodo de tiempo de interés, con picos de cobertura en los años 2009 y 2012, este último se prolongó hasta el año 2013. Al desagregar por periódico, la cobertura periodística del tema fue diferente, El Espectador publicó un número menor de noticias sobre tema de obesidad y en el año 2013, alcanzó su pico máximo de cobertura. Por su parte El Colombiano tuvo un comportamiento constante en la publicación anual de noticias y alcanzó su pico de cobertura en el año 2009 (Figura 1).

\section{Figura 1. Frecuencia relativa y tendencia en las noticias publicadas por periódico y por año entre} los años 2008-2013

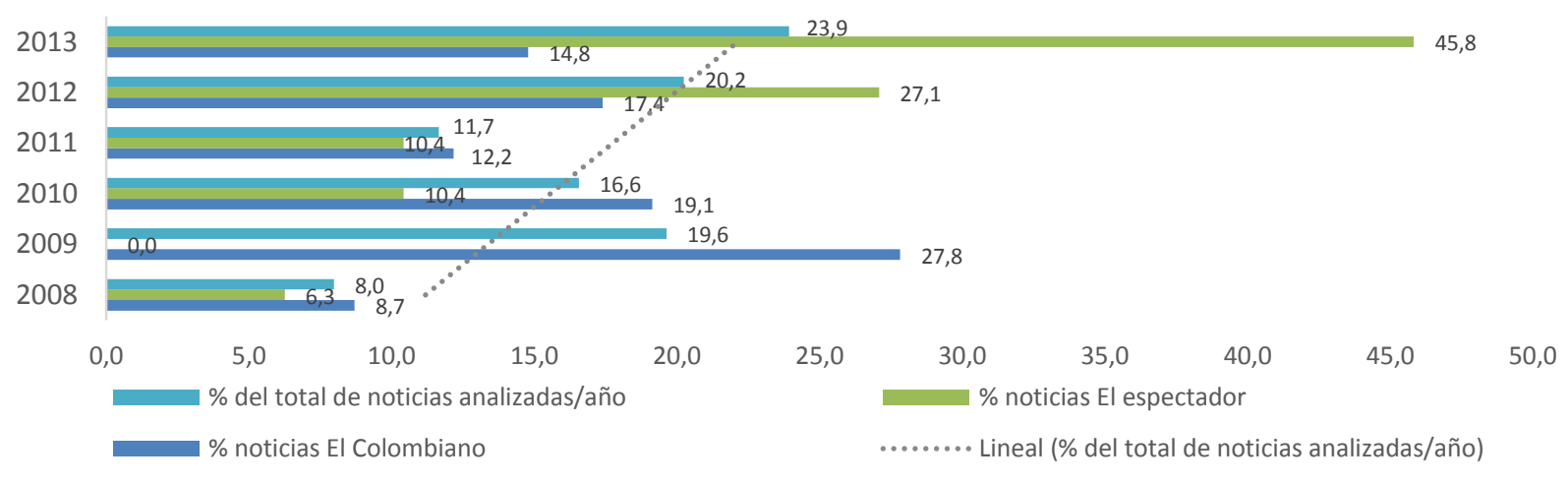


El 78,5\% de las noticias analizadas se publicó de lunes a viernes. Respecto a la sección del periódico, el 39,9\% se ubicó en la sección de noticias, 36,8\% sección salud, 11,0\% sección columnistas, 9,8\% otras secciones, $1,8 \%$ sección opinión, $0,6 \%$ sección economía, y en la sección de deportes no se publicó ninguna noticia sobre el tema (datos no mostrados). En cuanto a la población de referencia, se identificó que el 41,1\% aludió a la población infantil, $37,4 \%$ a población general, $35,0 \%$ adultos, $16,6 \%$ adolescentes y un $0,6 \%$ población de adultos mayores (datos no mostrados).

A propósito del tema los más tratados en su orden fueron: las estrategias para hacerle frente a la obesidad, la magnitud del problema y los resultados de investigaciones científicas; a su vez el tema menos tratado en las noticias analizadas fue la estética (Tabla 1).

Tabla 1. Distribución de las noticias por periódico según tema tratado

\begin{tabular}{lrrr}
\hline \multicolumn{1}{c}{ Temas } & $\begin{array}{c}\text { El } \\
\text { Espectador } \\
\mathbf{n = 4 8} \\
\mathbf{\%}\end{array}$ & $\begin{array}{c}\text { El } \\
\text { Colombiano } \\
\mathbf{n = 1 1 5} \\
\mathbf{\%}\end{array}$ & $\begin{array}{c}\text { total } \\
\mathbf{n = 1 6 3}\end{array}$ \\
\hline Estrategias & 58,3 & 64,3 & \multicolumn{1}{c}{62,6} \\
Magnitud & 56,3 & 53,9 & 54,6 \\
Resultados & & & \\
investigación & 29,2 & 40,9 & 37,4 \\
Recomendaciones & 20,8 & 25,2 & 23,9 \\
Denuncias & 16,7 & 20,9 & 19,6 \\
Bebidas & 20,8 & 14,8 & 16,6 \\
azucaradas & 31,3 & 8,7 & 15,3 \\
Comidas rápidas & 12,5 & 10,0 & 10,4 \\
Gasto Sanitario & 10,4 & 7,0 & 8,0 \\
Publicidad & 10,4 & 6,1 & 7,4 \\
Globalización & 2,1 & 2,6 & 2,5 \\
Estética & & & \\
\hline
\end{tabular}

Sobre las fuentes utilizadas para la construcción de las noticias, se encontró que las más consultadas fueron científico académicas, médicos o especialistas médicos, políticos, y datos epidemiológicos y de salud pública y las fuentes menos citadas las asociaciones profesionales, los nutricionistas dietistas y la industria farmacéutica (Tabla 2).

Tabla 2. Distribución de las noticias por periódico según la fuente principal

\begin{tabular}{|c|c|c|c|}
\hline Fuente & $\begin{array}{c}\text { El } \\
\text { Espectador } \\
\text { n=48 } \\
\% \\
\end{array}$ & $\begin{array}{c}\text { El } \\
\text { Colombiano } \\
\mathbf{n = 1 1 5} \\
\% \\
\end{array}$ & $\begin{array}{c}\text { Total } \\
\mathbf{n}=163 \\
\%\end{array}$ \\
\hline Científico académico & 47,9 & 40,0 & 42,3 \\
\hline $\begin{array}{l}\text { Médico o especialistas } \\
\text { médicos }\end{array}$ & 27,1 & 25,2 & 25,8 \\
\hline Político & 31,3 & 22,6 & 25,2 \\
\hline $\begin{array}{l}\text { Datos epidemiológicos } \\
\text { y de salud publica }\end{array}$ & 25,0 & 24,3 & 24,5 \\
\hline Periodista & 20,8 & 18,3 & 19,0 \\
\hline $\begin{array}{l}\text { Organizaciones } \\
\text { internacionales }\end{array}$ & 10,4 & 18,3 & 16,0 \\
\hline Jurídico judicial & 14,6 & 8,7 & 10,4 \\
\hline Industria de alimentos & 12,5 & 6,1 & 8,0 \\
\hline $\begin{array}{l}\text { Asociaciones } \\
\text { profesionales }\end{array}$ & 14,6 & 5,2 & 8,0 \\
\hline Nutricionistas dietistas & 6,3 & 7,0 & 6,7 \\
\hline Industria farmacéutica & 0,0 & 9,6 & 6,7 \\
\hline
\end{tabular}

Al cruzar las variables fuente de información Vs tema, se observó diferencia significativa cuando la fuente fue científico académica y el tema resultados de investigación $(76,8 \%)$.

Las asociaciones profesionales $(61,5 \%)$, la industria farmacéutica $(54,5 \%)$, los médicos y especialistas $(35,7 \%)$ fueron las más consultadas para la construcción de notas periodísticas sobre recomendaciones para el control y prevención de la obesidad $(\mathrm{p}<0,05)$. A su vez, se halló asociación entre la industria de alimentos y temas como denuncias $(46,2 \%)$, publicidad de alimentos $(38,5 \%)$ y bebidas azucaradas $(46,2 \%)$; mientras que su participación fue menor en temas como recomendaciones $(0,0 \%)$ y resultados de investigación $(7,7 \%)$ (Tabla 3). 
Tabla 3. Relación entre las variables fuente y tema

\begin{tabular}{|c|c|c|c|c|c|c|c|c|c|c|c|c|}
\hline & & 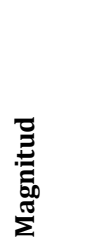 & 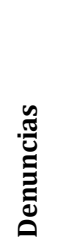 & 莺 & 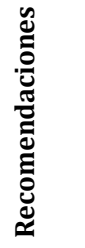 & 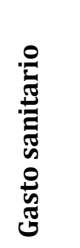 & 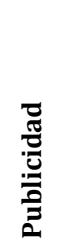 & 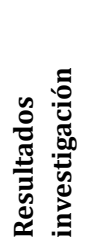 & 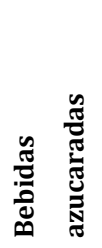 & 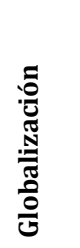 & 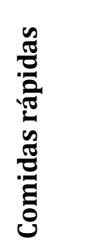 & 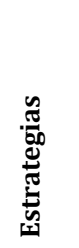 \\
\hline \multirow[t]{2}{*}{ Científico académico } & $\mathrm{Si}(\mathrm{n}=69)$ & 50,7 & 15,9 & 1,4 & 23,2 & 13,0 & 5,8 & $76,8 \dagger$ & $8,7 \dagger$ & 4,3 & $7,2 \dagger$ & $50,7 \dagger$ \\
\hline & No $(n=94)$ & 57,4 & 22,3 & 3,2 & 24,5 & 8,5 & 9,6 & 8,5 & 22,3 & 9,6 & 21,3 & 71,3 \\
\hline \multirow{2}{*}{$\begin{array}{l}\text { Médico o especialistas } \\
\text { médicos }\end{array}$} & $\mathrm{Si}(\mathrm{n}=42)$ & 57,1 & 26,2 & 0,0 & $35,7 \dagger$ & 4,8 & 9,5 & 45,2 & 14,3 & 9,5 & 11,9 & 61,9 \\
\hline & No $(n=121)$ & 53,7 & 17,4 & 3,3 & 19,8 & 12,4 & 7,4 & 34,7 & 17,4 & 6,6 & 16,5 & 62,8 \\
\hline \multirow[t]{2}{*}{ Nutricionista } & Si $(n=11)$ & 81,8 & 27,3 & 0,0 & 45,5 & 18,2 & 18,2 & 27,3 & 9,1 & 18,2 & 9,1 & 45,5 \\
\hline & No $(n=152)$ & 52,6 & 19,1 & 2,6 & 22,4 & 9,9 & 7,2 & 38,2 & 17,1 & 6,6 & 15,8 & 63,8 \\
\hline \multirow[t]{2}{*}{ Industria alimentos } & $\operatorname{Si}(n=13)$ & 61,5 & $46,2 *$ & 0,0 & $0,0 *$ & 15,4 & $38,5 *$ & $7,7 *$ & $46,2 *$ & 0,0 & 30,8 & 61,5 \\
\hline & No $(n=150)$ & 54,0 & 17,3 & 2,7 & 26,0 & 10,0 & 5,3 & 40,0 & 14,0 & 8,0 & 14,0 & 62,7 \\
\hline \multirow{4}{*}{$\begin{array}{l}\text { Asociaciones } \\
\text { profesionales } \\
\text { Industria farmacéutica }\end{array}$} & $\mathrm{Si}(\mathrm{n}=13)$ & 69,2 & 38,5 & 0,0 & $61,5 *$ & 7,7 & 7,7 & 30,8 & 7,7 & 0,0 & 23,1 & 76,9 \\
\hline & No $(n=150)$ & 53,3 & 18,0 & 2,7 & 20,7 & 10,7 & 8,0 & 38,0 & 17,3 & 8,0 & 14,7 & 61,3 \\
\hline & $\mathrm{Si}(\mathrm{n}=11)$ & $9,1^{*}$ & 18,2 & 0,0 & $54,5 *$ & 0,0 & 0,0 & 27,3 & 0,0 & 0,0 & 0,0 & $100 *$ \\
\hline & No $(n=152)$ & 57,9 & 19,7 & 2,6 & 21,7 & 11,2 & 8,6 & 38,2 & 17,8 & 7,9 & 16,4 & 59,9 \\
\hline \multirow[t]{2}{*}{ Jurídico Judicial } & si $(n=17)$ & 58,8 & 17,6 & 5,9 & 11,8 & 0,0 & $23,5 *$ & 29,4 & $47,1 *$ & 0,0 & $41,2 *$ & $100 \dagger$ \\
\hline & No $(n=146)$ & 54,1 & 19,9 & 2,1 & 25,3 & 11,6 & 6,2 & 38,4 & 13,0 & 8,2 & 12,3 & 58,2 \\
\hline \multirow[t]{2}{*}{ Político } & $\mathrm{Si}(\mathrm{n}=41)$ & $70,7 \dagger$ & 24,4 & 2,4 & 17,1 & 9,8 & 14,6 & $24,4 \dagger$ & $31,7 \dagger$ & 2,4 & $26,8 \dagger$ & $85,4 \dagger$ \\
\hline & No $(n=122)$ & 49,2 & 18,0 & 2,5 & 26,2 & 10,7 & 5,7 & 41,8 & 11,5 & 9,0 & 11,5 & 54,9 \\
\hline Organizaciones & $\operatorname{Si}(n=26)$ & $88,5 \dagger$ & 30,8 & 0,0 & 15,4 & $26,9 *$ & $23,1 *$ & 38,5 & 23,1 & 7,7 & 23,1 & 53,8 \\
\hline internacionales & No $(n=137)$ & 48,2 & 17,5 & 2,9 & 25,5 & 7,3 & 5,1 & 37,2 & 15,3 & 7,3 & 13,9 & 64,2 \\
\hline \multirow[t]{2}{*}{ Periodista } & $\mathrm{Si}(\mathrm{n}=31)$ & $35,5 \dagger$ & 19,4 & $9,7 *$ & 29,0 & 6,5 & 0,0 & $9,7 \dagger$ & 9,7 & 6,5 & 9,7 & 58,1 \\
\hline & No $(n=132)$ & 59,1 & 19,7 & 0,8 & 22,7 & 11,4 & 9,8 & 43,9 & 18,2 & 7,6 & 16,7 & 63,6 \\
\hline \multirow{2}{*}{$\begin{array}{l}\text { Datos epidemiológicos } \\
\text { y de salud publica }\end{array}$} & $\operatorname{Si}(n=40)$ & $95,0 \dagger$ & 22,5 & 0,0 & 27,5 & 17,5 & 5,0 & 32,5 & 15,0 & 10,0 & 12,5 & 62,5 \\
\hline & No $(n=123)$ & 41,5 & 18,7 & 3,3 & 22,8 & 8,1 & 8,9 & 39,0 & 17,1 & 6,5 & 16,3 & 62,6 \\
\hline
\end{tabular}

Nota: Los datos en números representan cifras porcentuales.

* Diferencias significativas con relación al no para cada tema según test de Fischer

† Diferencias significativas con relación al no para cada tema según $\mathrm{Chi}^{2}$ de Pearson

Cuando se empleó como fuente a los políticos, la mayoría de artículos hicieron referencia a la magnitud del problema de la obesidad $(70,7 \%)$, así mismo, cuando los temas fueron estrategias $(85,4 \%)$, bebidas azucaradas $(31,7 \%)$ y comidas rápidas $(26,8 \%) \mathrm{p}<0,05$. La fuente jurídica judicial se asoció con temas como estrategias $(100,0 \%)$, bebidas azucaradas $(47,1 \%)$, comidas rápidas $(41,2 \%)$ y publicidad $(23,5 \%)$. Por su parte, las organizaciones internacionales fueron el principal referente para los artículos sobre magnitud $(88,5 \%)$, gasto sanitario $(26,9 \%) \mathrm{y}$ publicidad $(23,1 \%)$. Los periodistas fueron las fuentes principales en temas sobre estética $(9,7 \%)$ (Tabla 3$)$.

En cuanto al cruce de variables población Vs tema, se hallaron diferencias significativas $(p<0,05)$ cuando el tema fue la magnitud del problema, sin embargo, este resultado no fue significativo en adultos mayores, sobre los cuales sólo se encontró una noticia. Con referencia a la población infantil, los temas publicidad $(16,4 \%)$ y comidas rápidas $(26,9 \%)$ tuvieron una mayor cobertura. Por su parte, el tema estrategias $(75,4 \%)$ fue tratado en mayor medida al referirse a la población en general (Tabla 4). 
Tabla 4. Relación entre las variables población y tema

\begin{tabular}{|c|c|c|c|c|c|c|c|c|c|c|c|c|}
\hline & & 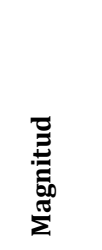 & 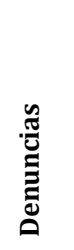 & 苞 & 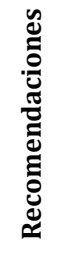 & 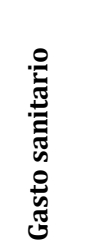 & 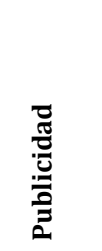 & 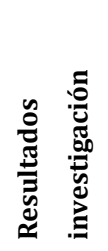 & 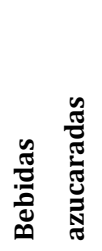 & 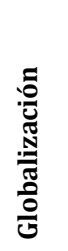 & 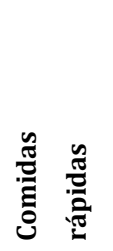 & 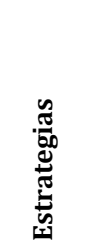 \\
\hline \multirow[t]{2}{*}{ Niños } & Si $(n=67)$ & $65,7 \dagger$ & 14,9 & 0,0 & 29,9 & 11,9 & $16,4 \dagger$ & 34,3 & 20,9 & 6,0 & $26,9 \dagger$ & 56,7 \\
\hline & No $(n=96)$ & 46,9 & 22,9 & 4,2 & 19,8 & 9,4 & 2,1 & 39,6 & 13,5 & 8,3 & 7,3 & 66,7 \\
\hline \multirow[t]{2}{*}{ Adolescentes } & Si $(n=27)$ & $81,5 \dagger$ & 14,8 & 0,0 & 22,2 & 14,8 & 14,8 & 25,9 & 22,2 & 11,1 & 25,9 & 59,3 \\
\hline & No $(n=136)$ & 49,3 & 20,6 & 2,9 & 24,3 & 9,6 & 6,6 & 39,7 & 15,4 & 6,6 & 13,2 & 63,2 \\
\hline \multirow[t]{2}{*}{ Adultos } & Si $(n=57)$ & $70,2 \dagger$ & 22,8 & 0,0 & 26,3 & 15,8 & 7,0 & 45,6 & 10,5 & 10,5 & 8,8 & $47,4 \dagger$ \\
\hline & No $(n=106)$ & 46,2 & 17,9 & 3,8 & 22,6 & 7,5 & 8,5 & 33,0 & 19,8 & 5,7 & 18,9 & 70,8 \\
\hline \multirow[t]{2}{*}{ Adultos mayores } & $\operatorname{Si}(n=1)$ & 0,0 & 0,0 & 0,0 & 0,0 & 100,0 & 0,0 & 100,0 & 0,0 & 0,0 & 0,0 & 100,0 \\
\hline & No $(n=162)$ & 54,9 & 19,8 & 2,5 & 24,1 & 9,9 & 8,0 & 37,0 & 16,7 & 7,4 & 15,4 & 62,3 \\
\hline \multirow[t]{2}{*}{ Población general } & Si $(n=61)$ & $36,1 \dagger$ & 19,7 & $6,6 *$ & 19,7 & 6,6 & 3,3 & 32,8 & 19,7 & 4,9 & 9,8 & $75,4 \dagger$ \\
\hline & No $(n=102)$ & 65,7 & 19,6 & 0,0 & 26,5 & 12,7 & 10,8 & 40,2 & 14,7 & 8,8 & 18,6 & 54,9 \\
\hline
\end{tabular}

\section{Discusión}

Durante el periodo de tiempo de interés del estudio, se observa una tendencia al aumento en el número de noticias sobre obesidad, al respecto los resultados evidencian dos picos de cobertura periodística, el año 2009 y 2013, los cuales coinciden con eventos importantes en la temática a nivel nacional, como lo son la promulgación de la Ley 1355 de $2009^{9}$ y la publicación de los resultados de la ENSIN 2010 en agosto de 20112. Aunque la frecuencia de publicación de noticias sobre obesidad en los dos periódicos fue diferente, estos concuerdan en los picos de cobertura.

El aumento de cobertura periodística denota el mayor interés de los medios de comunicación por este tema y cómo empieza a ser parte de la agenda mediática ${ }^{10} \mathrm{y}$, por tanto, la relevancia social de su abordaje. Un resultado similar fue obtenido por Ortiz et $a^{20}$, en la revisión de la cobertura periodística de la obesidad en prensa española (2000-2005) en el cual, los picos de cobertura coincidían con eventos importantes como la declaración de la obesidad como epidemia mundial, y la aplicación de la Estrategia para la Nutrición, Actividad Física y Prevención de la Obesidad en España (NAOS).

Debido a que la obesidad es un reconocido problema de salud pública a nivel mundial, el incremento de la cobertura periodística refleja la relevancia creciente del tema ${ }^{24}$, lo anterior se relaciona con el incremento en publicaciones sobre obesidad 25 , el continuo aumento en la prevalencia de esta condición $^{1}$ y un mayor entendimiento del impacto social de la obesidad ${ }^{26}$, todo lo anterior muestra la gran oportunidad de ejercer abogacía en los medios de comunicación como estrategia para movilizar la opinión frente a esta problemática.

Los diarios analizados publicaron más reportajes sobre obesidad de lunes a viernes, en los que normalmente el número de lectores es menor comparado con el fin de semana ${ }^{27}$, esto contrasta con el tipo de publicación, pues el mayor número de textos relacionados con el tema fueron publicados en la sección de noticias, que como su nombre lo indica es la utilizada para temas de actualidad y relevancia, seguida de la sección 
salud, resultado importante, pues los dos diarios analizados cuentan con un espacio específico para este tema, contrario a lo evidenciado en el análisis de los medios españoles, donde la salud no tiene una sección específica en la prensa ${ }^{20}$.

La ENSIN 2010 evidenció una prevalencia de exceso de peso del $65,7 \%$ en la población de 58 a 64 años (para mayores de 64 años no se cuenta con estadísticas de país) ${ }^{2}$, sin embargo sólo una noticia hizo referencia a los adultos mayores, situación que preocupa si se tiene en cuenta la proyección de crecimiento para el año 2020 del $42,1 \%$, para la población mayor de 60 años en Colombia ${ }^{28}$, por lo tanto es necesario incluirlos dentro de los diagnósticos de país y de las políticas de prevención y tratamiento de la obesidad.

En contraste, el $41,1 \%$ de las noticias hace referencia a la población infantil, y un 16,6\% a los adolescentes, poblaciones donde el sobrepeso y la obesidad crece con rapidez. Según la ENSIN 2010 la prevalencia de sobrepeso u obesidad en niños, niñas, y adolescentes de 5 a 17 años aumentó en un 25,9\% desde el año 2005 al 2010². Un resultado similar se encontró en el análisis de la prensa española, donde tres periódicos nacionales aumentaron los espacios dedicados a noticias sobre el sobrepeso y la obesidad infantil, en relación con el aumento de la tasa de estas condiciones en los niños y adolescentes de dicho país ${ }^{29}$.

En cuanto al tema con mayor cobertura periodística, los hallazgos de este estudio destacan las estrategias que tienen como fin promocionar la salud, prevenir el sobrepeso y la obesidad, o tratarla como proceso de enfermedad; las estrategias más presentadas fueron de tipo político-legal, donde las propuestas prioritarias giran en torno al incremento de impuestos a diferentes productos relacionados con la obesidad como lo son las bebidas azucaradas y la comida rápida. En contexto, estrategias impositivas, ya han sido aplicadas en países como México, donde a partir del primero de enero de 2014 se aplicó un impuesto de un peso mexicano por litro, a las bebidas con azúcares agregados ${ }^{30}$. Otros países como Brasil, Chile, Costa Rica,
México, Perú, Ecuador y Uruguay, cuentan con estrategias regulatorias para controlar la comercialización de alimentos obesogénicos que son vendidos en tiendas escolares ${ }^{31,32}$.

En este estudio, se destacó el uso de fuentes expertas sobre el tema y de los actores políticos para la construcción de las notas periodísticas; como lo hallado por Gearhart et al., que justifican el amplio uso de estas fuentes, debido a que proporcionan contexto, explicación y legitimación a la noticia ${ }^{26 .}$

Los hallazgos denotan la importancia de las fuentes científico académicas y los médicos o especialistas médicos, $(42,3 \%$ y $25,8 \%$ respectivamente) como referentes para la redacción de noticias sobre resultados de investigación y recomendaciones, acorde a lo encontrado por Ortiz et al., $(39,9 \% \text { y } 25,3 \%)^{20}$. Aunque los profesionales de la salud son una fuente frecuentemente utilizada, en este estudio los nutricionistas dietistas no están contemplados allí, por lo tanto, este profesional tiene como reto ejercer un papel más activo para lograr ser un líder de opinión en este tema que es de su competencia.

En el abordaje de la temática de publicidad, la fuente principal fueron las organizaciones internacionales, lo que pone de relieve la importancia del tema, situación que se refleja en el establecimiento por la OMS de una serie de recomendaciones dentro de las cuales se destaca la elaboración de políticas para el control de la publicidad dirigida a niños 33. A su vez, La Organización Panamericana de la Salud (OPS), propone una serie de recomendaciones dentro de las cuales se destaca la elaboración de políticas públicas para regular la publicidad de alimentos dirigidos a menores de edad ${ }^{34}$.

Este estudio logró evidenciar el aumento de cobertura del tema obesidad en prensa colombiana durante el periodo de interés, a la par que en el país se movilizan discusiones y se implementan estrategias para su prevención y control, lo cual muestra la congruencia entre la agenda mediática y la agenda política desde la promulgación de la ley de obesidad en el año 
2009. El abordaje periodístico de la obesidad en Colombia ha trascendido del tema magnitud del problema, al tema de las estrategias para controlar y combatir la obesidad, situación que favorece un papel movilizador de la opinión pública en torno a la búsqueda de acciones que puedan generar impacto positivo en la reducción de esta problemática.

Conflicto de intereses: Los autores declaran que en su conocimiento no existe conflicto de intereses.

\section{Referencias}

1. NCD Risk Factor Collaboration. Trends in adult bodymass index in 200 countries from 1975 to 2014: a pooled analysis of 1698 population-based measurement studies with 19.2 million participants. Lancet. 2016; 387:1377-96

2. Colombia. Ministerio de la Protección Social. Encuesta Nacional de la Situación Nutricional en Colombia 2010. Bogotá: Instituto Colombiano de Bienestar Familiar; 2011.

3. Fundación Colombiana de Obesidad. Guías colombianas para el manejo científico de la obesidad y sobrepeso [internet]. [Consultado 2015 Marzo 14]. Disponible en: http://academia.utp.edu.co/medicinadeportiva/files/2 012/04/GUIAS-COLOMBIANAS-PARA-MANEJOCIENTIFICO-OBESIDAD.pdf

4. Organización Mundial de la Salud. Global Status Report on noncommunicable diseases 2014. Suiza: OMS; 2014.

5. Organización Mundial de la Salud. Diez datos sobre la obesidad [internet]. [Consultado 2014 Marzo 10]. Disponible http://www.who.int/features/factfiles/obesity/es/

6. Wright SM, Aronne LJ. Causes of obesity. Abdom imaging [internet]. 2012; 37:730-732. [Consultado 2014 Marzo 11]. Disponible en: http://link.springer.com/article/10.1007/s00261-0129862-x\#page-1.

7. Rodríguez A, González B. El trasfondo económico de las intervenciones sanitarias en la prevención de la obesidad. Rev. Esp. Salud Pública [Internet]. 2009; 83(1): 25-41. Disponible [Consultado 2016 Jun 01] Disponible en: http://scielo.isciii.es/scielo.php?script=sci_arttext\&pid =S1135-57272009000100003\&lng=es.

8. Organización Mundial de la Salud. Estrategia mundial sobre régimen alimentario, actividad física y salud. Ginebra: OMS; 2004.

9. Colombia. Congreso de la República de Colombia. Ley 1355 de 2009 por medio de la cual se define la obesidad y las enfermedades crónicas no transmisibles asociadas a ésta como una prioridad de salud pública y se adoptan medidas para su control, atención y prevención. Bogotá: Congreso de la Republica; 2009.
10. Odriozola J. Cibermedios y agenda-setting: la configuración de la agenda mediática internacional. Estudios sobre el Mensaje Periodístico. 2012; 18(1): 157-171.

11. La iniciativa de comunicación y medios para el desarrollo de América Latina y el Caribe. Comunicación en salud: conceptos, teorías y experiencias [internet]. [Consultado 2014 Marzo 10]. Disponible en: http://www.comminit.com/la/drum_beat_52.html.

12. Healthy People 2020. Health Communication and Health Information Technology [internet]. [Consultado 2016 Junio 01]. Disponible en: https://www.healthypeople.gov/2020/topicsobjectives/topic/health-communication-and-healthinformation-technology

13. Lobo F. Políticas públicas para la promoción de la alimentación saludable y la prevención de la obesidad. Rev Esp Salud Pública. 2007; 81: 437-441.

14. Feo Acevedo C, Feo Istúriz O. Impacto de los medios de comunicación en la salud pública. Saúde em Debate [Internet]. 2013 Mar; 37(96):8 4-95. [Consultado 2014 Abril 07]. Disponible en: http://www.scielo.br/scielo.php?script=sci_arttext\&pi $\mathrm{d}=\mathrm{S} 0103-$

11042013000100010\&lng=es\&nrm=iso\&tlng=en

15. McCombs M. The agenda setting role of the mass media in the shaping of public opinion [internet]. [Consultado 2015 Marzo 10]. Disponible en: http://www.infoamerica.org/documentos_pdf/mccom bs01.pdf.

16. McCombs M. Setting the agenda the mass media and public opinion [Internet]. Cambridge: Polity Press; 2004. [Consultado 2015 Marzo 10]. Disponible en: http://books.google.com.co/books?id=hMxrVxROkHEC \&printsec=frontcover \&hl=es\&source=gbs_ge_summary _r\&cad $=0 \# v=$ onepage \& $\mathrm{q} \& \mathrm{f}=$ false.

17. Graber D.A. Mass media and Americans politics. 8 ed. [Internet]. SAGE; 2009. [Consultado 2015 Marzo 10]. Disponible http://books.google.com.co/books?id=m17havzYHQC\&printsec $=$ frontcover\&hl=es\&source $=$ gbs $_{-}$ ge_summary_r\&cad $=0 \# \mathrm{v}=$ onepage $\& \mathrm{q} \& \mathrm{f}=$ false.

18. Remesar X. ¿Cómo comunicamos? Las particulares relaciones entre científicos y medios de comunicación. Rev Esp Obes. 2006; 4(3): 131-133.

19. Asociación Colombiana de Investigación de Medios. Estudio General de Medios EGM Estructura de Medios 12011 Estratos 1 al 6 [Internet]. [Consultado 2014 Marzo 11]. Disponible en: http://www.acimcolombia.com/archivos/FILE_DOC_P UBLICACION/CIFRASEGM-JUNIO2011.pdf.

20. Ortiz-Barreda G, Vives-Cases C, Ortiz-Moncada R. La cobertura periodística de la obesidad en la prensa española (2000-2005). Perspect Nutr Humana. 2012; 14 (1):23-32.

21. Revuelta G. Salud y medios de comunicación en España. Gac Sanit. 2006; 20 (1): 203-208.

22. Asociación Colombiana de Investigación de Medios. Estudio General de Medios. Principales resultados segunda ola 2014. 
23. Monitoreo de Medios. Panorama del sector Prensa [Internet]. [Consultado 2016 Abril 06]. Disponible en: http://www.monitoreodemedios.co/panoramaprensa/

24. Weaver, D.H. Thoughts on agenda setting, framing, and priming. Journal of communication. 2007; 57 (1): 142147.

25. Scopus. Análisis de búsqueda obesidad por año (20002013) [Internet]. [Consultado 2016 Junio 01]. Disponible en: https://www.scopus.com/term/analyzer.uri?sid=B78D 93816C35DBFEC61CC3386034B66B.euC1gMODexYlPk Qec4u1Q\%3a10\&origin=resultslist\&src=s\&s=TITLEABS-KEY\%28obesity\%29\&sort=plf$\mathrm{f} \& \mathrm{sdt}=\mathrm{b} \& \mathrm{sot}=\mathrm{b} \& \mathrm{sl}=22 \&$ count $=334565 \&$ analyzeResults $=$ Analyze+results $\&$ txGid $=0$

26. Gearhart S, Craig C, Steed C. Network News Coverage of Obesity in Two Time Periods: An Analysis of Issues, Sources, and Frames. Health commun. 2012; 27:653662.

27. Rojo P. Extendiendo las marcas periodísticas en la era digital: hacia una producción informativa personalizada y dirigida a múltiples segmentos de público [internet]. [Consultado 2015 Marzo 10]. Disponible en: http://www.razonypalabra.org.mx/mundo/2005/agos to.html

28. Departamento Administrativo Nacional de Estadística de Colombia (DANE). Proyecciones nacionales y departamentales de población 2005 - 2020. Bogotá: DANE; Marzo 2010.

29. Westall D. La obesidad infantil en la prensa española. Estudios sobre el Mensaje Periodístico. 2011; 17(1): 225-239.

30. Instituto Nacional de Salud Pública de México. Resultados preliminares sobre los efectos del impuesto de un peso a bebidas azucaradas en México [internet]. [Consultado 2014 Noviembre 06]. Disponible en: http://www.insp.mx/epppo/blog/preliminaresbebidas-azucaradas.html.

31. OPS/FAO. Seminario experiencias exitosas en la prevención de obesidad infantil. Santiago; 2013. [internet]. [Consultado 2016 Junio 01]. Disponible en: http://www.paho.org/chi/index.php?option=com_doc man\&task=doc_view\&gid=126\&Itemid

32. Popkin BM, Adair LS, Wen S. Global nutrition transition and the pandemic of obesity in developing countries. Nutr Rev. 2012; 70(1):3-21.

33. OMS. Conjunto de recomendaciones sobre la promoción de alimentos y bebidas no alcohólicas dirigida a los niños. Ginebra; 2010.

34. OPS. Recomendaciones de la consulta de expertos de la Organización Panamericana de la Salud sobre la promoción y publicidad de alimentos y bebidas no alcohólicas dirigida a los niños en la Región de las Américas. Washington, D.C. 2011. 